\title{
ON MAXI-QUASIPROJECTIVE MODULES
}

\author{
MANABU HARADA \\ (Received 19 May 1982; revised 12 August 1982) \\ Communicated by R. Lidl
}

\begin{abstract}
We have defined a mini-injective module and given some structures of self mini-injective rings and certain relationships between such rings and QF-rings in [8] and [9].

In this short note we shall study the modules dual to mini-injective modules, which we call maxi-quasiprojective modules. We shall give a characterization and some structures, in terms of the above modules, of those rings whose every injective module has the lifting property of direct decompositions modulo the Jacobian radical (see [5], [6] and [7]). Furthermore, we shall show that the above rings are closely related to QF-rings (see [8] and [9]).
\end{abstract}

1980 Mathematics subject classification (Amer. Math. Soc.): 16 A 36.

Throughout this note, we assume that a ring $R$ contains an identity and every module is a unitary right $R$-module. We always assume that $R$ is a right artinian ring unless otherwise stated. However, some of the first part of this note is valid without this assumption.

A part of this paper was prepared when the author visited University of Sydney in 1981. The author would like to express his thanks to Professor M. Kelly and his colleagues for their kind hospitality, and to the referee for the useful suggestion to revise the paper.

\section{Maxi-quasiprojective modules}

Let $M$ be an $R$-module. We denote the Jacobson radical of $M$ by $J(M)$. We put $\bar{M}=M / J(M)$. If $N$ is a direct summand of $M, N / J(N)$ may be regarded as an $R$-submodule of $\bar{M}$. Hence $\bar{N}=N / J(N) \subseteq \bar{M}$. An $R$-module $T$ is called hollow if 
$J(T)$ is a unique maximal submodule of $T$. Since $R$ is artinian, $\bar{M}$ is semi-simple. Let $\bar{M}=\Sigma_{I} \oplus N_{\alpha}$, where the $N_{\alpha}$ are simple. If there exists a direct decomposition $\Sigma_{I} \oplus M_{\alpha}$ of $M$ with $\vec{M}_{\alpha}=N_{\alpha}$ for each $\alpha \in I$, we say that the direct decomposition $\bar{M}=\Sigma_{I} \oplus N_{\alpha}$ is lifted to $M$. If $M$ has the above property for any direct decomposition of $\bar{M}$, we say that $M$ has the lifting property of direct decompositions of $\bar{M}$ [6]. In this case $M=\Sigma_{I} \oplus M_{\alpha}$ and the $M_{\alpha}$ are hollow modules. Hence $M_{\alpha} \approx e_{\alpha} R / e_{\alpha} A$, where the $e_{\alpha}$ is a primitive idempotent and $A$ is a right ideal in $R$.

It is well known that every projective module has the lifting property of direct decompositions modulo the radical [12]. We note that if $N_{\alpha} \approx N_{\beta}$ for each pair $\alpha$, $\beta$, every direct summand of $\bar{M}$ is of the form $\Sigma_{K} \oplus N_{\delta}(K \subseteq I)$ and so $M$ has trivially the lifting property of direct decompositions of $\bar{M}$. In order to avoid this trivial case, we assume that

(\#) each $N_{\alpha}$ is isomorphic to another $N_{\beta}$ [7].

Now, we shall define a new class of modules. For any maximal submodule $N$ of $M$, we consider a diagram

$$
\begin{aligned}
& 0 \rightarrow N \rightarrow M \quad \stackrel{\nu}{\rightarrow} M / N \quad \rightarrow 0 \\
& \text { ז } \uparrow f \\
& \text { M }
\end{aligned}
$$

where $\nu$ is the natural epimorphism. If, for any $f$ in $\operatorname{Hom}_{R}(M, M / N)$, there exists an element $h$ in $\operatorname{End}_{R}(M)$ which makes the above diagram commute, we say that $M$ is a maxi-quasiprojective module. It is clear that every quasi-projective module is maxi-quasiprojective and the converse is not true in general. For instance, let $R$ be a local algebra over a field $K$ such that $R / J(R) \approx K$ and let $A$ be a right ideal of $R$. Then $R / A$ is maxi-quasiprojective, but not quasi-projective, provided that $A$ is not a two-sided ideal (see Remarks 2 and 3 below).

Let $N$ and $N^{\prime}$ be two maximal submodules of $M$. Then the definition above is equivalent to the fact:

$$
\left(\operatorname{Hom}_{R}(M, M) \supseteq\right) \operatorname{Hom}_{R}(M, M)^{*} \rightarrow \operatorname{Hom}_{R}\left(M / N, M / N^{\prime}\right)
$$

is an epimorphism via natural epimorphisms $\nu$ and $\nu^{\prime}$, where $\operatorname{Hom}_{R}(M, M)^{*}=$ $\left\{f \in \operatorname{Hom}_{R}(M, M) \mid f(N) \subseteq N^{\prime}\right\}$.

We put $S=\operatorname{End}_{R}(M), \bar{S}=\operatorname{End}_{R}(\bar{M})$ and $J_{0}(S)=\operatorname{Hom}_{R}(M, J(M))$. Then we have the natural monomorphism $\theta: S / J_{0}(S) \rightarrow \bar{S}$ (see [7]).

THEOREM 1. Let $M$ be an $R$-module. Assume $M=\Sigma_{I} \oplus M_{\alpha}$ and the $M_{\alpha}$ are completely indecomposable; that is, $\operatorname{End}_{R}\left(M_{\alpha}\right)^{*}$ is local. We further assume ( $\left.\#\right)$. Then the following conditions are equivalent:

1) $\theta$ is an epimorphism. 
2) $M$ is maxi-quasiprojective and each $M_{\alpha}$ is hollow.

3) $M$ has the lifting property of direct decompositions of $\bar{M}$.

Proof. 1) $\rightarrow 2$ ). We note that every element in $S$ (resp. $\bar{S}$ ) is expressed as a column summable matrix with entries $f_{\alpha \beta}$, where the $f_{\alpha \beta}$ are elements in $\operatorname{Hom}_{R}\left(M_{\beta}, M_{\alpha}\right)$ (resp. $\operatorname{Hom}_{R}\left(\bar{M}_{\beta}, \bar{M}_{\alpha}\right)$ ). Hence it is clear that $\theta$ induces an epimorphism $\theta_{\alpha}: \operatorname{End}_{R}\left(M_{\alpha}\right) \rightarrow \operatorname{End}_{R}\left(\bar{M}_{\alpha}\right)$. Since $\operatorname{End}_{R}\left(M_{\alpha}\right)$ is local, so is End $_{R}\left(\bar{M}_{\alpha}\right)$. Furthermore, $\bar{M}_{\alpha}$ is semi-simple and so $\bar{M}_{\alpha}$ is simple. Therefore $M_{\alpha}$ is hollow. Let $N_{1}$ and $N_{2}$ be two maximal submodules of $M$. Then $N_{i} \supseteq J(M) \approx$ $\bar{M} / N_{1}$ is a direct summand of $\bar{M}$. Accordingly, $M$ is maxi-quasiprojective.

2) $\rightarrow 3$ ). Since $M_{\alpha}$ is hollow, $J\left(M_{\alpha}\right) \oplus \Sigma_{\beta \neq \alpha} \oplus M_{\beta}$ is a maximal submodule of $M$. Hence $\operatorname{Hom}_{R}\left(M_{\alpha}, M_{\beta}\right) \rightarrow \operatorname{Hom}_{R}\left(\bar{M}_{\alpha}, \bar{M}_{\beta}\right)$ is an epimorphism for $\alpha, \beta \in I$. We assume $\bar{M}_{\alpha} \approx \bar{M}_{\beta}$. Then $M_{\alpha}, M_{\beta}$ being hollow, there exist epimorphisms $f$ : $M_{\alpha} \rightarrow M_{\beta}, g: M_{\beta} \rightarrow M_{\alpha}$ by the above. Since $R$ is artinian and so the $M_{\alpha}$ are of finite length, $M_{\alpha} \approx M_{\beta}$. Hence $\left\{M_{\alpha}\right\}_{I}$ is (semi-) $T$-nilpotent (see [11]). Therefore $M$ has the lifting property of direct decompositions of $M$ by [7], Corollary 1 to Theorem 2.

3) $\rightarrow 1$ ). This is clear from [7], Theorem 2.

Theorem 2 (the dual to [8], Theorem 3). Let $R$ be a right artinian ring. Then the following two conditions are equivalent:

1) Every injective $E$ has the lifting property of direct decompositions of $\bar{E}$.

2) An injective cogenerator is maxi-quasiprojective and a direct sum of hollow submodules: that is, right $\mathrm{QF}-2^{*}[5]$.

Proof. Every injective is a direct sum of completely indecomposable modules. Hence the theorem is clear from Theorem 1 and [7], Theorem 2 and its remark (note that we do not use the assumption (\$) for the implication 2 ) $\rightarrow 3$ ) in the proof).

REMARKS. 1. We can define an essentially quasi-projective module as the dual to uni-injective [8], when we replace a maximal submodule by an essential submodule. We note that if $M$ is essentially quasi-projective, $M$ is maxi-quasiprojective and if $M$ is uniform and essentially quasi-projective, $M$ is quasi-projective.

2. We take the ring defined in [8], Example 2. Let $L \supseteq K$ be two field satisfying the following conditions: $[L: K]=2$ and there exists an isomorphism $\sigma$ of $L$ onto $K$. Put $R=L \oplus L u$ a vector space over $L$. We define a product on $R$ as $\left(x_{1}+x_{2} u\right)\left(y_{1}+y_{2} u\right)=x_{1} y_{1}+\left(x_{2} \sigma\left(y_{1}\right)+x_{1} y_{2}\right) u$, where the $x_{i}$ and the $y_{i}$ are in $L$. Then $R$ is mini-injective as a right $R$-module [8]. $R^{*}=\operatorname{Hom}_{K}(R, K)$ as right $K$-modules is a left $R-K$ bimodule and $R^{* *} \approx R$ as right $R-K$ bimodules. Then $R^{*}$ 
is an indecomposable and left $R$-maxi-quasiprojective module, which is not hollow.

3. Let $K$ be a field and

$$
R=\left(\begin{array}{lll}
K & 0 & K \\
0 & K & K \\
0 & 0 & K
\end{array}\right)
$$

Put $e=e_{11}$ and $f=e_{22}$. Then $e R \approx f R$ and $\mathrm{S}(e R) \approx \mathrm{S}(f R)(=S)$, where $\mathrm{S}()$ means the socle of ( ). Put $M=(e R \oplus f R) / S^{\prime}$, where $S^{\prime}=\{s+s \mid s \in S\}$. Then $M$ is maxi-quasiprojective, since $\bar{M}=e \bar{R} \oplus f \bar{R}$ and $e \bar{R} \approx f \bar{R}$ and $M$ is an indecomposable module, which is not hollow (see [1]).

\section{Lifting property of injectives}

We shall study the right artinian rings whose every injective module has the lifting property of direct decompositions modulo the radical (see [5]).

First we shall give the dual to 1 ) of Theorem 5 in [8].

LEMMA 3. Let $M_{1}$ and $M_{2}$ be indecomposable modules of finite length.

1) If $M=M_{1} \oplus M_{2}$ is maxi-quasiprojective, and $M_{1} \approx M_{2}$, then no simple submodule in $\bar{M}_{1}$ is isomorphic to a submodule in $\bar{M}_{2}$.

2) If $M_{1}$ is maxi-quasiprojective, and $M_{1} / N_{1} \approx M_{1} / N_{2}$ for maximal submodules $N_{i}$ in $M_{1}$, then there exists an automorphism $f$ of $M_{1}$ with $f\left(N_{1}\right)=N_{2}$.

Proof. 1) Assume there exists a maximal submodule $N_{i}$ of $M_{i}$ such that $M_{1} / N_{1} \approx M_{2} / N_{2}$. Then there exist $f_{1}$ in $\operatorname{Hom}_{R}\left(M_{1}, M_{2}\right)$ and $f_{2}$ in $\operatorname{Hom}_{R}\left(M_{2}, M_{1}\right)$ which induce the given isomorphism and satisfy $f_{1}\left(N_{1}\right) \subseteq N_{2}$ and $f_{2}\left(N_{2}\right) \subseteq N_{1}$. Put $h=f_{2} f_{1} \in \operatorname{End}_{E}\left(M_{1}\right)$. Then $h\left(N_{1}\right) \subseteq N_{1}$ and $M_{1}=h\left(M_{1}\right)+N_{1}$. If $h$ is not an isomorphism, $h$ is nilpotent, for $M_{1}$ has finite composition length. Hence $M_{1}=$ $h^{n}\left(M_{1}\right)+N_{1}=N_{1}$ for some $n$, which is a contradiction. Therefore $h$ is an isomorphism and $f_{i}$ is also an isomorphism, a contradiction.

2) If we apply the above argument for $M_{1}=M_{2}$, we have 2).

LEMMA 4. Let $M_{1}$ be an indecomposable module of finite length. We put $M=M_{1}^{(I)}$ a direct sum of $|I|$-copies of $M_{1}$. We assume that $M_{1}$ is maxi-quasiprojective and $N$ a maximal submodule of $M$. Then there exists a decomposition $M=\Sigma_{I} \oplus M_{\alpha}^{\prime}$ of $M$ such that $N=M_{1} \oplus \Sigma_{I-\{1\}} \oplus M_{\alpha}^{\prime}$, where $M_{\alpha}^{\prime} \approx M_{1}$ for all $\alpha \in I$ and $N_{1}$ is a maximal submodule of $M_{1}^{\prime}$, where $|I|$ means the cardinal of $I$. 
Proof. We shall show that $N$ contains a non-zero direct summand of $M$ if $|I| \geqslant 2$. Since $N$ is a maximal submodule, $N \supseteq J(M)$. We denote $M / J(M)$ by $\bar{M}$ (note that, for a submodule $K$ of $M, \bar{K}=(K+J(M)) / J(M)$ ). Let $M=\Sigma_{I} \oplus M_{\alpha}$, and put $M_{\alpha} \cap N=N_{\alpha}$. If $M_{\alpha} \subseteq N$ for some $\alpha$, we are done. Hence we may assume $M=N+M_{\alpha}$ for any $\alpha \in I$. Then $M / N=\left(N+M_{\alpha}\right) / N \approx M_{\alpha} / N_{\alpha}$ and so $N_{\alpha}$ is a maximal submodule of $M_{\alpha}$. Now, since $\bar{M}_{\alpha}$ is semi-simple,

(1) $\bar{M}_{\alpha}=\bar{N}_{\alpha} \oplus \bar{A}_{\alpha}$, where $J\left(M_{\alpha}\right) \subseteq A_{\alpha} \subseteq M_{\alpha}$ and $\bar{A}_{\alpha}$ is a simple submodule.

Since $N \supseteq \Sigma_{I} \oplus N_{\alpha} \supseteq J(M), \bar{N}=\Sigma_{I} \oplus \bar{N}_{\alpha} \oplus \bar{N}_{0}$ and $\bar{N}_{0} \neq 0$, for $N$ is maximal and $|I| \geqslant 2$. Let $\bar{N}_{0}^{*}$ be a simple submodule of $\bar{N}_{0} ; N_{0}^{*} \supseteq J(M)$. We take the decomposition

$$
\bar{M}=\left(\Sigma_{I} \oplus \bar{N}_{\alpha}\right) \oplus\left(\Sigma_{I} \oplus \bar{A}_{\alpha}\right)
$$

Let $p$ and $p_{\alpha}$ be the projection of $\bar{M}$ onto $\Sigma_{I} \oplus \bar{N}_{\alpha}$ and $\bar{A}_{\alpha}$, respectively. Since $\bar{N}_{0}^{*}$ is simple, there exists a finite subset $I^{\prime}$ of $I$ such that $p_{\alpha}\left(\bar{N}_{0}^{*}\right)=\overline{A_{\alpha}}$ for $\alpha \in I^{\prime}$ and $p_{\beta}\left(\bar{N}_{0}^{*}\right)=0$ for $\beta \in I-I^{\prime}$. We may assume $I^{\prime}=\{1,2, \ldots, n\}$. Hence there exists a set of isomorphisms $g_{i}: \bar{A}_{1} \rightarrow \bar{A}_{i}(i \geqslant 2)$ such that $\bar{N}_{0}^{*}=\left\{p(n)+a_{1}+g_{2}\left(a_{1}\right)\right.$ $\left.+\cdots+g_{n}\left(a_{1}\right) \mid n \in \bar{N}_{0}^{*}, a_{1}=p_{1}(n) \in \bar{A}_{1}\right\}$. On the other hand, there exists $f_{i}$ : $M_{1} \rightarrow M_{i}$ such that $f_{i}\left(N_{1}\right)=N_{i}$ and $f_{i}$ induces $g_{i}$ on $\overline{A_{1}}$ by Lemma 3 . We put $M_{1}(f)=\left\{m_{1}+f_{2}\left(m_{1}\right)+\cdots+f_{n}\left(m_{1}\right) \mid m_{1} \in M_{1}\right\}$. Then it is clear that $M_{1}(f) \approx$ $M_{1}$ and $M=M_{1}(f) \oplus \Sigma_{I-\{1\}} \oplus M_{\alpha}$. Let $\bar{m}_{1}=\bar{n}_{1}+\bar{a}_{1}$, where $\bar{n}_{1} \in \bar{N}_{1}$ and $\bar{a}_{1} \in \bar{A}_{1}$. Then there exists $n$ in $N_{0}^{*}$ such that $\bar{n}=p(\bar{n})+\bar{a}_{1}+g_{2}\left(\bar{a}_{1}\right)$ $+\cdots+g_{n}\left(\bar{a}_{1}\right)$. Hence $\overline{m_{1}+f_{2}\left(m_{1}\right)+\cdots+f_{n}\left(m_{1}\right)}=\bar{n}_{1}+f_{2}\left(\bar{n}_{1}\right)$ $+\cdots+f_{n}\left(\bar{n}_{1}\right)+\bar{a}_{1}+f_{2}\left(\bar{a}_{1}\right)+\cdots+f_{n}\left(\bar{a}_{1}\right)=\bar{n}_{1}+f_{2}\left(\bar{n}_{1}\right)+\cdots+f_{n}\left(\bar{n}_{1}\right)+\bar{n}-$ $p(\bar{n}) \in \Sigma_{1} \oplus \bar{N}_{\alpha} \oplus N_{0}^{*} \subseteq \bar{N}$, and so $M_{1}(f) \subseteq N$. Let $\mathbf{F}$ be the set of all direct sums of indecomposable modules $K_{\alpha}$ isomorphic to $M_{1}$, which are contained in $N$ and are locally direct summands of $M$; that is, any finite sum of the $K_{\alpha}$ is a direct summand of $M$. Then $F$ is non-empty by the above, and we can find a maximal member in $F$ with respect to the relation to the members of direct components by Zorn's lemma, say $\Sigma_{J} \oplus M_{\alpha}^{\prime}(\subseteq N)$. Since $M_{1}$ has the finite length, $\left\{M_{\alpha}\right\}_{I}$ is a semi- $T$-nilpotent set [11]. Hence $\Sigma_{J} \oplus M_{\alpha}^{\prime}$ is a direct summand of $M$ by [11], Theorem, say $M=\Sigma_{J} \oplus M_{\alpha}^{\prime} \oplus M^{*}$. Hence $N=\Sigma_{J} \oplus M_{\alpha}^{\prime} \oplus\left(N \cap M^{*}\right)$ and $N \cap$ $M^{*}$ is a maximal submodule of $M^{*}$. $M^{*}$ is also a direct sum of submodules isomorphic to $M_{1}$ by [16]. Therefore $|I-J|=1$ by the above and the maximality of $\Sigma_{J} \oplus M_{\alpha}^{\prime}$.

We assume that an $R$-module is a direct sum of indecomposable modules $M_{\alpha}$ of finite length. Then we can rearrange this decomposition as follows:

$$
M \approx \Sigma_{\alpha \in I} \oplus M_{\alpha}^{\left(J_{\alpha}\right)}, \quad M_{\alpha} \approx M_{\beta} \text { if } \alpha \neq \beta
$$


TheOREM 5. Let $M$ be as in (3). Then $M$ is maxi-quasiprojective if and only if the $M_{\alpha}$ are maxi-quasiprojective for all $\alpha$ and no simple submodule in $\bar{M}_{\alpha}$ is isomorphic to a simple submodule in $\bar{M}_{\beta}$ if $\alpha \neq \beta$ (and hence $|I|$ is finite).

Proof. Assume $M$ is maxi-quasiprojective. Then so is any direct summand of $M$ by the definition. Hence we have the property above. Since $R$ is artinian, $I$ is finite. Let $N$ be a maximal submodule in $M$. By the assumption, $\bar{M}_{\alpha}^{\left(J_{\alpha}\right)}$ is a direct sum of homogeneous components in $\bar{M}$. Hence $\bar{N}=\bar{N}_{1} \oplus \Sigma_{\alpha \neq 1} \oplus \bar{M}_{\alpha}^{\left(J_{\alpha}\right)}$ for some homogeneous component $M_{1}^{\left(J_{1}\right)}$, where $N_{1}$ is a maximal submodule in $M_{1}^{\left(J_{1}\right)}$, and so $M / N \approx M_{1}^{\left(J_{1}\right)} / N_{1}$. We take another maximal submodule $N^{\prime}$ of $M$ such that $M / N \approx M / N^{\prime}$. Then as above, we obtain $\overline{N^{\prime}}=\overline{N_{1}^{\prime}} \oplus \Sigma_{\alpha \neq 1} \oplus \bar{M}_{\alpha}^{\left(J_{\alpha}\right)}$, where $N_{1}^{\prime}$ is a maximal submodule of $M_{1}^{\left(J_{1}\right)}$. Now we obtain two decompositions of $M_{1}^{\left(J_{1}\right)}$ by Lemma 4:

$$
\begin{aligned}
& M_{1}^{\left(J_{1}\right)}=M_{1}^{\prime\left(J_{1}-1\right)} \oplus M_{1}^{\prime} \supseteq N=M_{1}^{\prime\left(J_{1}-1\right)} \oplus N_{1}^{\prime} \text { and } \\
& M_{1}^{\left(J_{1}\right)}=M_{1}^{\prime \prime\left(J_{1}-1\right)} \oplus M_{1}^{\prime \prime} \supseteq N^{\prime}=M_{1}^{\prime \prime\left(J_{1}-1\right)} \oplus N_{1}^{\prime \prime},
\end{aligned}
$$

where $N_{1}^{\prime} \subset M_{1}^{\prime}$ and $N_{1}^{\prime \prime} \subset M_{1}^{\prime \prime}$ and $M_{1} \approx M_{1}^{\prime} \approx M_{1}^{\prime \prime}$. Hence we obtain an automorphism $f$ of $M_{1}^{\left(J_{1}\right)}$ by the assumption, which induces the given isomorphism between $M_{1}^{\prime} / N_{1}^{\prime}$ and $M_{1}^{\prime \prime} / N_{1}^{\prime \prime}$. Therefore $M$ is maxi-quasiprojective.

Corollary 1. Let $M$ be as above and let $N$ be a submodule of $M$ containing $J(M)$. We assume that $M$ is maxi-quasiprojective and each $M_{\alpha}$ is cyclic hollow. Then there exists a decomposition of $M$ such that $M=\Sigma_{I} \oplus M_{\beta}^{\prime} \supset N=\Sigma_{I_{1}} \oplus M_{\beta}^{\prime}$ $\oplus \Sigma_{I_{2}} \oplus J\left(M_{\gamma}^{\prime}\right): I=I_{1} \cup I_{2}$ and the $M_{\alpha}^{\prime}$ are indecomposable. Let $N^{\prime}$ be another submodule of $M$ containing $J(M)$. If $M / N \approx M / N^{\prime}$, there exists an automorphism $f$ of $M$ which induces the above isomorphism and $f(N)=N^{\prime}$.

Proof. We take the same argument as the proof of Lemma 4. Since $M_{\alpha}$ is hollow, $\overline{A_{\alpha}}$ is either simple or zero. Hence, if $N \neq J(M), N$ contains a non-zero direct summand of $M$ from the method after (1) in the proof of Lemma 4. We can use the same argument for the remainder.

Corollary 2 (the dual to [8], Theorem 5). Let $E=\sum_{J=1}^{n} \oplus E_{i}$ be a minimal injective cogenerator with $E_{i}$ indecomposable. We assume that $E$ is finitely generated and maxi-quasiprojective. Then

1) All simple submodules in $\bar{E}_{i}$ are isomorphic to one another and are not isomorphic to any one in $\bar{E}_{j}$ for $i \neq j$. 
2) If $\bar{E}_{i}$ is simple for all $i$, every primitive idempotent $e$ in $R$ is non-small [3]; that is, $e R$ is not a small submodule in the injective envelope $\mathrm{E}(e R)$ of $e R$ and $R$ contains all simple submodules up to isomorphism and $\mathrm{r}(J) \subseteq 1(J)$, where $J=J(R), 1(J)=$ $\{x \in R \mid x J=0\}$ and $\mathbf{r}(J)=\{x \in R \mid J x=0\}$.

Proof. 1) Since $E$ is a minimal cogenerator, $E_{i} \approx E_{j}$ for $i \neq j$ and $\sum_{i=1}^{n} \oplus E_{i}$ contains all simple $R$-modules up to isomorphism by Lemma 3 . Hence we obtain 1) from Lemma 3.

2) If $\bar{E}_{i}$ is simple for all $i, R$ is right QF-2* [5]. Hence we obtain the non-isomorphic representative set of indecomposable and injective modules $\left\{e_{1} R / e_{1} A_{1}, e_{2} R / e_{2} A_{2}, \ldots, e_{n} R / e_{n} A_{n}\right\}$ from Theorem 2 and [5], Theorem 3, where $\left\{e_{i}\right\}$ is the set of mutually orthogonal and non-isomorphic primtive idempotents and $\left\{A_{i}\right\}$ is a set of right ideals. We assume that $e_{i}$ is small. Then $\tilde{E}=\mathrm{E}\left(e_{i} R\right) \supset \mathrm{J}(\tilde{E}) \supseteq e_{i} R \supseteq e_{i} A_{i}$. Since $e_{i} R / e_{i} A_{i}$ is injective, $\tilde{E} / e_{i} A_{i}$ contains a direct summand $e_{i} R / e_{i} A_{i}$ contained in $J\left(\tilde{E} / e_{i} A_{i}\right)$, which is a contradiction. Let $\mathrm{E}(R) \approx \Sigma_{K} \oplus\left(e_{j} R / e_{j} A_{j}\right)^{m_{j}}$, where $\left(e_{j} R / e_{j} A_{j}\right)^{m_{j}}$ is a direct sum of $m_{j}$-copies of $e_{j} R / e_{j} A_{j}$, and $K \subseteq\{1,2, \ldots, n\}$. Since $e_{i}$ is non-small, $K$ contains $i$. Hence $\mathrm{E}(R)$ is a cogenerator, and so $R$ contains all simple modules up to isomorphism. We may assume that $R \subseteq \Sigma_{i=1}^{k} \oplus e_{i} R / e_{i} A_{i}$ and $\mathrm{r}(J) \subseteq \sum_{i=1}^{k} \oplus \bar{a}_{i} \mathrm{r}(J)$, where $a_{i} \in$ $e_{i} R, \bar{a}_{i}=a_{i}+e_{i} A_{i}$ and $e_{i}$ may equal $e_{j}$ for some $j$. We assume that $a_{1} \mathrm{r}(J) \nsubseteq$ the socle $\mathrm{S}\left(e_{1} R / e_{1} A_{1}\right)$ of $e_{1} R / e_{1} A_{1}$. Since $e_{1} R / e_{1} A_{1}$ is uniform, $\bar{a} \mathrm{r}(J) \supseteq S_{1}=$ $\mathrm{S}\left(e_{1} R / e_{1} A_{1}\right)$. $\mathrm{E}(R)$ being an injective cogenerator, there exists $f$ in $\operatorname{Hom}_{R}\left(e_{1} R / e_{1} A_{1}, e_{i} R / e_{i} A_{i}\right)$ for some $i$ such that $f\left(S_{1}\right)=0$ and $f\left(\bar{a}_{1} \mathrm{r}(J)\right) \neq 0 . f$ is given by the left-sided multiplication of an element $b$ in $e_{i} R e_{1}$. Since $f\left(S_{1}\right)=0$, $f$ is not an isomorphism. Hence $b \in e_{i} J e_{1}$ by the construction of $e_{i} R / e_{i} A_{i}$ (note that $e_{i} R / e_{i} A_{i} \nsim e_{j} R / e_{j} A_{j}$ if $\left.i \neq j\right) . f\left(\bar{a}_{1} \mathrm{r}(J)\right)=b \bar{a}_{1} \mathrm{r}(J) \subseteq \bar{e}_{i} J \mathrm{r}(J)=0$, a contradiction. Therefore $\bar{a}_{1} \mathrm{r}(J) \subseteq S_{1}$ and $\bar{a}_{1} \mathrm{r}(J) J \subseteq S_{1} J=0$. Similarly, we have $\bar{a}_{i} \mathrm{r}(J) J=0$ for all $i$ and so $\mathrm{r}(J) J=0$. Thus, $\mathrm{r}(J) \subseteq 1(J)$.

The following theorem is the dual to [8], Theorem 13.

THEOREM 6. Let $R$ be a right artinian ring. Then the following conditions are equivalent:

1) $R$ is a QF-ring.

2) $R$ is right $\mathrm{QF}-2$ and $\mathrm{QF}-2 *$ and a minimal injective cogenerator is maxi-quasiprojective (see Theorem 9 below).

3) Every injective $E$ has the lifting property of direct decompositions of $\bar{E}$ and $\mathrm{l}(J) \subseteq \mathrm{r}(J)$.

4) Every injective $R$-module and every injective left $R$-module have the lifting property of direct decompositions modulo the radical. 
Proof. 1) $\rightarrow 2$ ). Since $R$ is an injective cogenerator and a projective module as a right $R$-module by [2], we obtain 2).

$2) \rightarrow 1$ ). Let $e$ be a primitive idempotent. $R$ being QF-2 and QF-2*, $\mathrm{E}(e R)$ is hollow. Hence $\mathrm{E}(e R)=e R$ by 2 ) and Corollary 2 to Theorem 5. Therefore $R$ is self-injective (see the proof of [8], Theorem 13).

1) $\rightarrow 3$ ) and 4). Since every injective module is projective by [2] and $1(J)=\mathrm{r}(J)$ by [15], we have 3 ) and 4 ).

$3) \rightarrow 1$ ). We know from 3 ) and Theorem 2 that minimal injective cogenerator are maxi-quasiprojective and $R$ is a QF-2* ring. Hence we shall use the same notation as in the proof of Corollary 2 to Theorem 5. Since $R \subseteq \mathrm{E}(R) \approx$ $\sum_{j=1}^{n} \oplus\left(e_{j} R / e_{j} A_{j}\right)^{m_{j}}, \sum_{i=1}^{n} \oplus e R / e_{i} A_{i}$ is faithful. We shall show that $e_{1} A_{1} \cap$ $\mathrm{r}(J)=0$. We assume $e_{1} A_{1} \cap \mathrm{r}(J) \neq 0$ and take a non-zero element $x$ in $e_{1} A_{1} \cap$ $\mathrm{r}(J)$. Then $e_{i} R x=e_{i} R e_{1} x \subseteq J x=0$ if $i \neq 1$. Hence $e_{1} R x \nsubseteq e_{1} A_{1}$. Let $y$ be an element in $e_{1} R e_{1}$ such that $y x \notin e_{1} A_{1}$. Since $x \in \mathrm{r}(J), y \notin e_{1} J e_{1}: e_{1} R / e_{1} A_{1}$ being maxi-quasiprojective and $y$ inducing an element in $\operatorname{End}_{R}\left(e_{1} R / e_{1} J\right)$, there exists an element $z$ in $e_{1} R e_{1}$ such that $y-z \in e_{1} J e_{1}$ and $z\left(e_{1} A_{1}\right) \subseteq e_{1} A_{1}$ by Lemma 3. Hence $y z=z x \in e_{1} A_{1}$, which is a contradiction. Similarly, $e_{i} A_{i} \cap \operatorname{r}(J)$ $=0$ for all $i$. Now, $1(J) \subseteq \mathrm{r}(J)$ and $1(J)$ is an essential right ideal in $R$. Hence $e_{i} A_{i}=0$ for all $i$, and so $R=\mathrm{E}(R)$.

COROLLARY 1. Let $R$ be a right artinian ring. Then $R$ is a QF-ring if and only if every injective $E$ and every projective $P$ have the lifting and extending property of direct decompositions of $\bar{E}$ and $\mathrm{S}(P)$, respectively. Furthermore, if $1(J)=\mathrm{r}(J)$ ( for example, $J^{2}=0$ or $R$ is commutative), we can replace the two conditions above by either one.

Proof. This is clear from Theorem 6 and [8], Theorem 5.

As is well known, $R$ is a QF-ring if and only if $R$ is self-injective as a right $R$-module. However, $R$ is actually quasi-injective as a right $R$-module from the definition of quasi-injective, and so $R$ is injective as a right $R$-module by Baer's criterion. Hence the concept dual to the above is the following: A (minimal) injective cogenerator is quasi-projective. Thus we have the following corollary.

COROLlary 2. Let $R$ be as above. Then $R$ is a QF-ring if and only if the minimal injective cogenerator is quasi-projective.

Proof. We assume that the minimal injective cogenerator is quasi-projective. Then every injective is quasi-projective by [10] and the proof of [4], Proposition 2.4. Put $E=(R)$. Then $E \approx \sum_{i=1}^{k} \oplus e_{i} R / e_{i} A_{i}$, where the $e_{i}$ are primitive 
idempotents, the $A_{i}$ are right ideals and $e_{j} R e_{i} e_{i} A_{i} \subseteq e_{j} A_{j}$ from the proof of [6], Corollary 3 in page 790. We may assume that $R \subseteq \sum_{i=1}^{k} \oplus e_{i} R / e_{i} A_{i}$ as a right $R$-module and $R$ is basic (see [13] and [14]). We note, from Corollary 2 to Theorem 5 , that the set $\left\{e_{i}\right\}$ contains the set of all non-isomorphic primitive idempotents. Let $1=\sum \tilde{a}_{i}$, where $a_{i}=e_{i} a_{i}$ and $\bar{a}_{i}=a_{i}+e_{i} A_{i}$. Then $J=\mathrm{J}(R) \subseteq$ $\Sigma \oplus \bar{a}_{i} J$. We shall show $\bar{a}_{j} J e 1(J)=0$ for all $i$ and $j$. Then, since $1(J)=$ $\Sigma_{K} \oplus e_{i} \mid(J)$, where $K \subseteq\{1,2, \ldots, k\}, 1(J) \subseteq \mathrm{r}(J)$. We note that $e_{j} R e_{i}=$ $\operatorname{Hom}_{R}\left(e_{i} R, e_{j} R\right)$ and each element in $e_{j} R e_{i}$ induces an element in $\operatorname{Hom}_{R}\left(e_{i} R / e_{i} A_{i}, e_{j} R / e_{j} A_{j}\right)$ from the above. Now, $\bar{a}_{1} J e_{1} 1(J)=\bar{e}_{1}\left(a_{1} J e_{1} 1(J)\right)=$ $\left(e_{1} a_{1} J e_{1}\right)\left(\bar{e}_{1} l(J)\right)$, where we obtain this equality by regarding $e_{1} a_{1} J e_{1} \subseteq$ $\operatorname{End}_{R}\left(e_{1} R / e_{1} A_{1}\right)$. Since $1(J)$ is semi-simple, so is $e_{1} l(J)$. Further $e_{1} a_{1} J e_{1} \subset$ $\mathrm{J}\left(\operatorname{End}_{R}\left(e_{1} R\right)\right)$ and each element in $\mathrm{J}\left(\operatorname{End}_{R}\left(e_{1} R\right)\right)$ induces an element in $\mathrm{J}\left(\operatorname{End}_{R}\left(e_{i} R / e_{1} A_{1}\right)\right)$. Hence $\left(e_{1} a_{1} J e_{1}\right)\left(\bar{e}_{1} 1(J)\right)=0$, for $e_{1} R / e_{1} A_{1}$ is uniform. Next we consider $e_{1} a_{1} J e_{2} 1(J)$. If $e_{2} R \approx e_{1} R$, then we have $\bar{e}_{1} a_{1} J e_{2} 1(J)=0$ from the above (note $e_{1} R / e_{1} A_{1} \approx e_{2} R / e_{2} A_{2}$ ). We assume $e_{2} R \approx e_{1} R$. Case 1: $e_{2} 1(J) \subseteq$ $e_{2} A_{2}$. Then $e_{1} A_{1} \supseteq e_{1} \operatorname{Rel}(J) \supseteq e_{1} a_{1} J e_{2} \mathrm{l}(J)$, since $e_{1} \operatorname{Re}_{2} e_{2} A_{2} \subseteq e_{1} A_{1}$. Hence $\bar{a} J e_{2} 1(J)=0$. Case 2: $e_{2} \mathrm{l}(J) \nsubseteq e_{2} A_{2}$ and $e_{1} a_{1} J e_{2} \mathrm{l}(J) \nsubseteq e_{1} A_{1}$. Then $e_{1} R / e_{1} A_{1}$ and $e_{2} R / e_{2} A_{2}$ contain the simple module isomorphic to $\bar{e}_{2} l(J)$, which is a contradiction. Hence, if $e_{2} 1(J) \nsubseteq e_{2} A_{2}, e_{1} a_{1} J e_{2} l(J) \subseteq e_{1} A_{1}$. Therefore $\bar{a}_{1} J e_{2} 1(J)$ $=0$. Similarly, $a_{i} J e_{j} l(J)=0$, and so $1(J) \subseteq \mathrm{r}(J)$. Since quasi-projective is maxi-quasiprojective, we have the corollary from Theorems 2 and 6.

Finally, we take an algebra. Let $K$ be a field and let $R$ be a $K$-algebra of finite dimension. In this case we note that we have the duality functor $\operatorname{Hom}_{K}(-, K)=$ $(-)^{*}$. Then every injective right $R$-module $E$ has the lifting property of direct decompositions of $\bar{E}$ if and only if every projective left $R$-module has the extending property of direct decompositions of the socle; namely, $R$ is left mini-injective and so $R$ is a QF-algebra by [9] (we note that we may restrict ourselves to the cases where every module is finitely generated by [7]). Therefore the following theorem is clear from the above and [9], Theorem 1. We shall give the dual proof for the sake of completeness.

THEOREM 7. Let $R$ be an algebra over a field $K$ with $[R: K]$ finite. Then the following conditions are equivalent:

1) $R$ is a QF-ring.

2) A minimal injective cogenerator is maxi-quasiprojective.

3) $R$ is a right self mini-injective ring.

Proof. 1) $\rightarrow$ 3). This is clear from [2]. 
$3) \rightarrow 2$ ). Since $R^{*}$ is an injective cogenerator as a left $R$-module, we obtain 2) for the left $R$-modules.

2 ) $\rightarrow 1$ ). We may assume that $R$ is basic and we use the same notations above. Since $R$ is an algebra of finite dimension, every indecomposable injective is finitely generated and isomorphic to $\left(R e_{i}\right)^{*}$. We denote $\left(R e_{i}\right)^{*}$ by $E_{i}$. Then $S_{i}=\operatorname{End}_{R}\left(E_{i}\right)$ is anti-isomorphic to $e_{i} R e_{i}$. Let $N_{i}$ be a maximal submodule of $E_{i}$ and put $\tilde{S}_{i}=\left\{x \in S_{i} \mid x\left(N_{i}\right) \subseteq N_{i}\right\}$. Since $N_{i}=\left(R e_{i} / T_{i}\right)^{*}$ for some minimal left ideal $T_{i}$ of $R e_{i}$ and $T_{i} \mathrm{~J}(R)=0$ by Corollary 2 to Theorem $5, \mathrm{~J}\left(S_{i}\right) N_{i} \subseteq N_{i}$, and so $\mathrm{J}\left(S_{i}\right) \subseteq \tilde{S}_{i}$. Then $\operatorname{End}_{R}\left(E_{i} / N_{i}\right)=\tilde{S}_{i} / \mathrm{J}\left(S_{i}\right)$ from 2). Hence $\operatorname{End}_{R}\left(E_{i} / N_{i}\right)$ is antiisomorphic to a $K$-subfield of $\overline{e_{i} R e_{i}}$. We put $E_{i} / N_{i} \approx \overline{e_{i^{\prime}} R}$. Then $\left[\overline{e_{i}, R e_{i}}: K\right] \leqslant\left[\overline{e_{i} R e_{i}}: K\right]$. Thus we obtain a chain of idempotents $\left\{e_{1}, e_{2}, \ldots, e_{i}, \ldots\right\}$ such that $E_{i}=\left(R e_{i}\right)^{*}$ and $E_{i}$ contains a maximal submodule $N_{i}$ with $E_{i} / N_{i} \approx \overline{e_{i+1} R}$. If $e_{i} R \approx e_{i+k} R$ for some $i$ and $k, E_{i-1} \approx E_{i+k-1}$ by Lemma 3 . Hence $e_{i-1} \approx e_{i+k-1}$. We know from this fact that the mapping: $i \rightarrow i^{\prime}$ gives us a permutation of $\{1,2, \ldots, n\}$, where $\Sigma_{i=1}^{n} \oplus(R e)^{*}$ is a minimal injective cogenerator. Hence $\left[\overline{e_{i^{\prime}} R e_{i^{\prime}}}: K\right]=\left[\overline{e_{i} \operatorname{Re}_{i}}: K\right]$. Let $N_{1}$ and $N_{2}$ be two maximal submodules of $E_{i}$. Then $E_{i} / N_{1} \approx E_{i} / N_{2}$ by Corollary 2 to Theorem 5 . Hence there exists an automorphism $x$ of $E_{i}$ such that $x\left(N_{1}\right)=N_{2}$ by Lemma 3. On the other hand, $S_{i}=\tilde{S}_{i}$ from the argument above. Hence $N_{2}=x\left(N_{1}\right) \subseteq N_{1}$, and so $N_{1}$ is a unique maximal submodule of $E_{i}$. Therefore $R$ is right QF-2*. Accordingly, every injective $E$ has the lifting property of direct decompositions of $E$ by Theorem 2 . Then we obtain a non-isomorphic representative set of indecomposable injectives $\left\{e_{1} R / e_{1} A_{1}, e_{2} R / e_{2} A_{2}, \ldots, e_{n} R / e_{n} A_{n}\right\}$ by [5], Theorem 3 and [6], Theorem 3 . Hence $\left\{\left(e_{i} R / e_{i} A_{i}\right)^{*}\right\}_{1}^{n}$ is a non-isomorphic representative set of indecomposable and projective left $R$-modules. Therefore $\sum_{i=1}^{n} \oplus\left(e_{i} R / e_{i} A_{i}\right)^{*} \approx R$ as left $R$-modules. Accordingly, $[R: K]=\sum_{i=1}^{n}\left[\left(e_{i} R / e_{i} A_{i}\right)^{*}: K\right]=\sum_{i=1}^{n}\left[e_{i} R / e_{i} A_{i}: K\right]$. Hence $e_{i} A_{i}=0$ for all $i$, and so $R$ is self-injective.

\section{Self mini-injective rings}

We shall add a characterization of right QF-2 and self mini-injective rings.

THEOREM 8. Let $R$ be a right artinian and basic ring. Then $R$ is a right QF-2 and self mini-injective ring if and only if $1(J)=R u=u R$ for some $u$ in $R$.

Proof. Let $R=\sum_{i=1}^{n} \oplus e_{i} R$ be as above. We assume that $R$ is a right QF-2

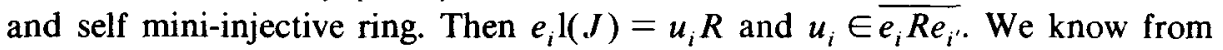
[8], Theorem 3 that $\mathrm{l}(J) \subseteq \mathrm{r}(J)$. Therefore, since $u_{i} R$ is a unique minimal right ideal, $R u_{i} \subseteq u_{i} R . \overline{e_{i^{\prime}} R e_{i^{\prime}}}$ being a division ring, a mapping: $u_{i} \rightarrow u_{i} r\left(r \in \overline{e_{i^{\prime}} R e_{i^{\prime}}}\right)$ is 
extendable to an element in $\operatorname{Hom}_{R}\left(u_{i} R, u_{i} R\right)$. Then, since $R$ is right self mini-injective, there exists an element $x$ in $e_{i} R e_{i}$ with $x u_{i}=u_{i} r$. Therefore $R u_{i}=u_{i} R$. Put $u=\sum_{i=1}^{n} u_{i}$. Then $e_{i} u=u e_{i^{\prime}}=u_{i}$. Hence $u R=\sum_{i=1}^{n} \oplus u_{i} R=\sum_{i=1}^{n} \oplus R u_{i}=$ $R u=1(J)$. Conversely, we assume $1(J)=u R=R u$. Then $1(J)$ is a homomorphic image of $R / J$ as right $R$-modules. Hence $u R \approx R / J$ from the composition length. Therefore $1(J)=\sum_{i=1}^{n} \oplus e_{i} 1(J), e_{i} 1(J)$ is a unique minimal right ideal, and so $R$ is right QF-2. Furthermore, $e_{i} \mathrm{l}(J) \ngtr e_{j} \mathrm{l}(J)$ if $i \neq j$. Hence $e_{i} 1(J)$ is a two-sided ideal, and so $1(J)=u R=R u$ implies $R e_{i} u=e_{i} u R=e_{i} l(J)$. Therefore $R$ is a right self mini-injective, since End ${ }_{R}\left(e_{i} u R\right)=\overline{e_{i} R e_{i}}$ as above.

THEOREM 9. Let $R$ be a right artinian ring. Then $R$ is a QF-ring if and only if $R$ is a right $\mathrm{QF}-2, \mathrm{QF}-2^{*}$ and self mini-injective ring.

Proof. We assume that $R$ satisfies the second condition of the theorem. We may assume that $R$ is basic. Let $R=\sum_{i=1}^{n} \oplus e_{i} R$, where the $e_{i}$ are primitive idempotents and $e_{i} R \approx e_{j} R$ if $i \neq j$. Since $R$ is QF-2 and QF-2*, $E\left(e_{i} R\right) \approx$ $e_{j} R / e_{j} A$ for some $j$ and some right ideal $A$. Then we have the diagram

$$
\begin{gathered}
e_{i} R \\
\downarrow i \\
e_{j} R \stackrel{\nu}{\rightarrow} e_{j} R / e_{j} A \rightarrow 0
\end{gathered}
$$

where $i$ is the inclusion and $\nu$ is the natural epimorphism. Since $e_{i} R$ is projective, there exists $f: e_{i} R \rightarrow e_{j} R$ such that $i=\nu f$. $i$ being a monomorphism, $f$ is the same. Hence $S\left(e_{i} R\right) \approx \mathrm{S}\left(e_{j} R\right)$ by the assumption. Therefore $i=j$ by [8], Theorem 5 . The fact that $e_{i} R \subset e_{i} R / e_{i} A$ implies $e_{i} A=0$. Hence $R$ is self-injective.

\section{References}

[1] H. Asashiba and T. Sumioka, 'On Krull-Schmidt's theorem and the indecomposability of amalgamated sums,' to appear.

[2] S. Eilenberg and T. Nakayama, 'On the dimension of modules and algebras II,' Nagoya Math. J. 9 (1956), 1-16.

[3] M. Harada, 'Non-small modules and non-cosmall modules,' Proc. of Ring Theory 1978, pp. 669-689 (Antwerp, Marcel Dekker, New York).

[4] M. Harada, 'Note on quasi-injective modules,' Osaka J. Math. 2 (1965), 351-356.

[5] M. Harada, 'On one-sided QF-2 rings I,' Osaka J. Math. 17 (1980), 421-431.

[6] M. Harada, 'On lifting property on direct sums of hollow modules,' Osaka J. Math. 17 (1980), 783-791.

[7] M. Harada, 'On modules with lifting properties,' Osaka J. Math. 19 (1982), 189-201.

[8] M. Harada, 'Self mini-injective rings', Osaka J. Math. 19 (1982), 587-597.

[9] M. Harada, 'A characterization of QF-rings,' to appear. 
[10] J. P. Jans and L. E. Wu, 'On quasi-projectives,' Illnois J. Math. 11 (1967), 439-448.

[11] H. Kanbara, 'Note on Krull-Remak-Schmidt-Azumaya's theorem,' Osaka J. Math. 9 (1972), 409-413.

[12] E. Mares, 'Semi-perfect modules,' Math. Z. 83 (1963), 347-360.

[13] K. Morita, 'Duality for modules and its applications to the theory of rings with minimum conditions,' Sci. Rep. Tokyo Kyoiku Daigaku Sect. A (1958), 83-142.

[14] M. Oshima, 'Notes on basic rings', Math. J. Okayama Univ. 2 (1952-53), 103-110.

[15] T. Nakayama, 'On Frobenius algebras II,' Ann. of Math. 42 (1941), 1-21.

[16] R. B. Warfield, Jr., 'Decompositions of injective modules,' Pacific J. Math. 31 (1969), 263-276.

\section{Osaka City University}

Sumiyoshi-Ku, Osaka 558

Japan 